\title{
Une nouvelle approche ontologique et statistique des construits et troubles mentaux : introduction à la psychiatrie des "networks"
}

\author{
Giovanni Briganti, M.D. \\ Unité d'épidémiologie, biostatistique et recherche clinique \\ École de Santé Publique, Université libre de Bruxelles \\ Route de Lennik 808, 1070 Anderlecht \\ giovanni.briganti@hotmail.com \\ Paul Linkowski, M.D., Ph.D. \\ Unité d'épidémiologie, biostatistique et recherche clinique \\ École de Santé Publique, Université libre de Bruxelles \\ Route de Lennik 808, 1070 Anderlecht
}

\begin{abstract}
Résumé
L'approche des "networks" connaît un succès de plus en plus important en psychiatrie et neurosciences computationnelles. Cette nouvelle méthodologie, issue de la translation de modèles de physique statistique en médecine, accorde une nouvelle vue ontologique sur les troubles mentaux, en les définissant comme des systèmes complexes émergeant de l'interconnexion entre différents symptômes. Cet article propose au clinicien une introduction tant théorique que méthodologique au potentiel dont dispose cette approche pour l'amélioration du diagnostic et traitement des maladies psychiatriques, en fournissant quelques exemples d'application explorée en recherche clinique.
\end{abstract}

Mots-clés networks $\cdot$ ontologie $\cdot$ biostatistique $\cdot$ psychométrie $\cdot$ psychiatrie

\section{Introduction}

Le mot "symptôme" est défini par le dictionnaire Larousse comme un "phénomène subjectif qui traduit les états morbides et qui est lié aux troubles fonctionnels ou lésionnels qui le déterminent". En pratique psychiatrique courante, les plaintes reportées par un patient au clinicien le prenant en charge ont été catégorisées comme "symptôme", définition exemplifiée dans les manuels diagnostiques de référence, tel l'ICD-10 ou le DSM-5. En médecine somatique, la présence d'un symptôme implique la présence d'une maladie sous-jacente, due à une étiologie uni- ou multi-factorielle identifiée le plus souvent.

En psychiatrie, cependant, le concept de "maladies mentales", bien qu'encore fort représenté dans le terrain médico-légal (nous pensons notamment à la loi belge du 26 juin 1990 relative à la mise en observation des malades mentaux), laisse progressivement la place au concept de "trouble mental", tel que décrit par le DSM-V (1) et faisant référence à un "syndrome". Le syndrome est à son tour défini comme un "ensemble de plusieurs symptômes ou signes en rapport avec un état pathologique donné et permettant, par leur groupement, d'orienter le diagnostic" : il s'agit dès lors d'un écosystème d'entités détectées par le clinicien ou déclarées à ce dernier; cet écosystème porte un sens empirique, mais pour une raison inconnue. En effet, pour la plupart des pathologies psychiatriques, l'approche étiopathogénique classique utilisée en recherche médicale s'est révélée infructueuse (2) : en psychiatrie, nous pouvons rarement identifier une cause qui, complètement éliminée, fasse disparaître l'entièreté de la présentation symptomatologique du patient, tel l'effet qu'une administration d'antibiotique aurait sur une infection causée par un germe particulier.

Pourtant, la psychométrie classique (le champ commun à la psychiatrie et à la psychologie qui vise à mesurer les entités mentales, tant normales que pathologiques) dispose à ce jour d'une très large majorité d'outils 
PsyChiATRIE DES "NETWORKS"

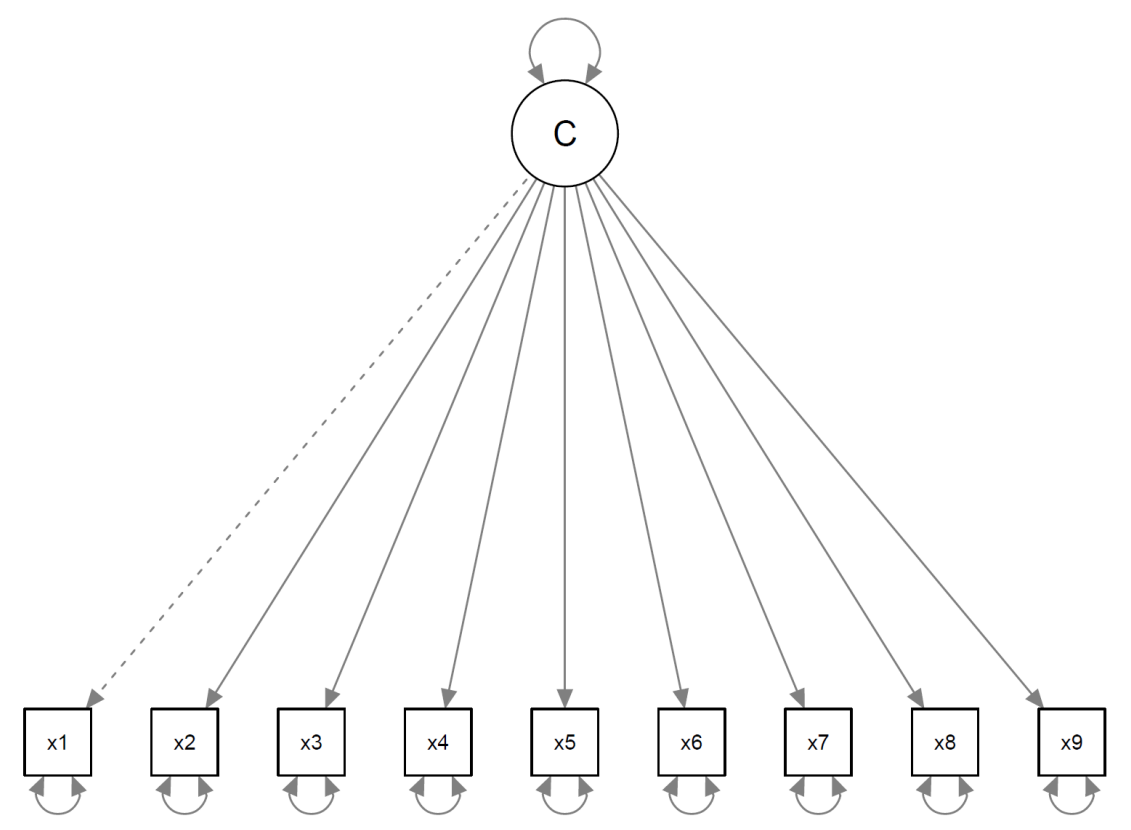

Figure 1 - Modèle de la cause commune. Une variable latente (C) cause les 9 symptômes.

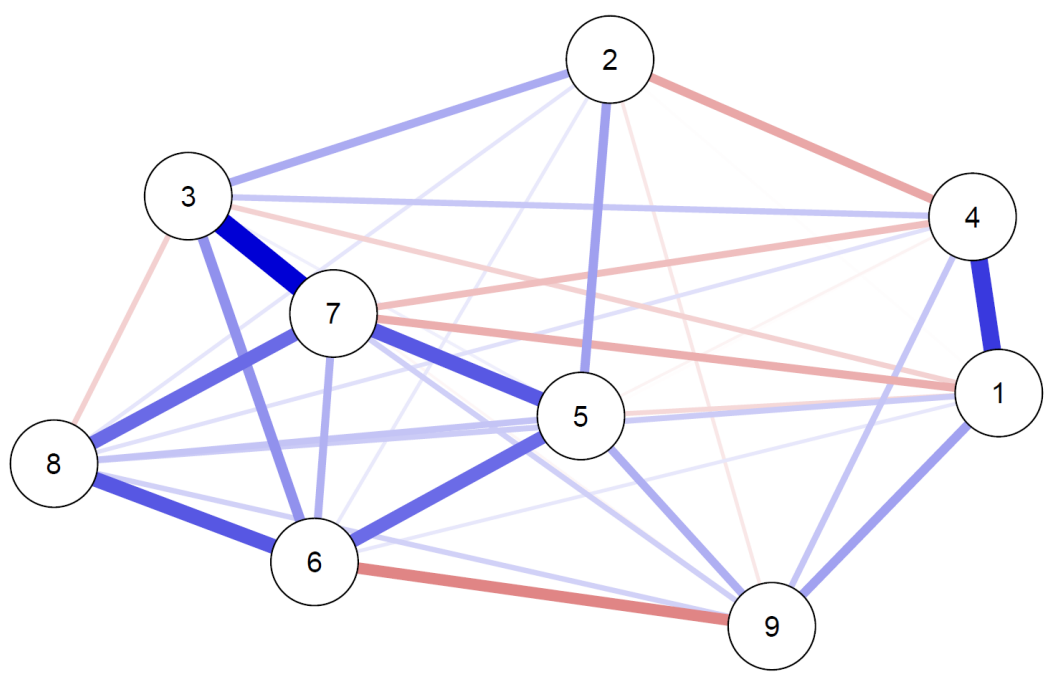

Figure 2 - Modèle des networks. Les variables (noeuds) interagissent via des connexions (bords). Les liens positifs sont colorés en bleu, les liens négatifs sont colorés en rouge. L'épaisseur d'un bord dénote son intensité (poids).

de mesure qui conçoivent les troubles mentaux comme étant la conséquence d'une cause commune. Cette approche, qui a été fort utilisée ces dernières décennies et bien consolidée dans la littérature scientifique est connue sous le nom de "modèle des variables latentes" (3). Selon le modèle des variables latentes, les symptômes rencontrés en clinique sont des éléments pouvant être mesurées, mais ne constituant qu'un reflet passif de la pathologie en question; telle une toux lors d'une pneumonie, le symptôme psychiatrique ne représente pas une composante qui cause la pathologie, mais est l'effet mesuré de la pathologie même.

Il y a une décennie, certains travaux ont avancé l'hypothèse qu'une cause centrale pour les troubles mentaux ne peut pas être retrouvée car il n'existe pas de cause centrale; au lieu d'être causés par une entité invisible, 
Psychiatrie DES "NETWORKS"

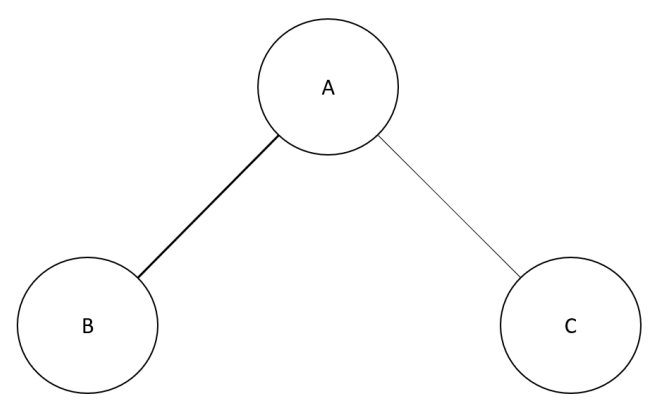

FIgURE 3 - Un network non dirigé à trois noeuds (A, B, et C). Les trois noeuds sont connectés par deux bords, A-B, et A-C. A-B est plus épais que A-C ; il possède un poids plus important.

les symptômes psychiatriques se causent mutuellement (4). Par exemple, si un patient possède des ruminations anxieuses, il pourra présenter des troubles du sommeil et il sera dès lors plus fatigué; la fatigue va générer un certain stress, qui va à son tour augmenter les ruminations. Avec une telle activation par rétroaction, l'état mental du patient peut dégénérer, jusqu'à ce qu'il puisse être défini comme un trouble mental. Cette hypothèse a été formellement définie comme la théorie "network" des troubles mentaux" (5) et a été appliquée dans de domaines différents de la psychiatrie et la psychologie clinique, comme le trouble du stress post-traumatique (6), l'empathie (7), la personnalité narcissique (8), la dépression (9), l'alexithymie (10), l'estime de soi (11), l'autisme (12), et la résilience (13). D'un point de vue mathématique, le modèle de la cause commune (illustré en figure 11 est équivalent au modèle des networks, dont un exemple est indiqué en figure 2

De récents travaux ont pu proposer un modèle intégratif combinant l'approche des variables latentes avec l'approche network : dans cette approche, appelée la psychométrie network généralisée (14) les symptômes peuvent être causées par différentes variables latentes et interagir entre eux. Cette approche s'est révélée utile pour simplifier l'analyse des networks basés sur des échelles psychométriques, où différentes dimensions sont représentées par le biais de plusieurs items redondants. Une fois la redondance limitée en représentant les symptômes par les variables latentes qu'il mesurent par exemple un domaine particulier d'un construits mentaux, tel l'apport de l'opinion de soi dans le construit de l'estime de soi (11), une interaction entre les différentes variables latentes est observée. Certains travaux ont pu démontrer que des nouveaux outils, tels que l'analyse graphique exploratoire (15), spécifiquement conçue pour la détection de domaines dans des networks (traduire "facteurs" dans l'approche par variables latentes) est capable de détecter le bon nombre de dimensions dans un échantillon; si le nombre détecté n'est pas le même, un nouveau modèle est proposé qui convient mieux aux données de l'échantillon.

Le but de ce travail est de proposer une introduction générale à la méthodologie des networks au médecin clinicien en apportant une attention particulière sur les implications que le développement de cette approche peut apporter au diagnostic et traitement des troubles mentaux. Nous y détaillons les défis, les opportunités et les critiques principales qu'ont été décrites dans la littérature de ce domaine, qui a rapidement évolué dans cette dernière décennie. Nous allons premièrement décrire la structure et composition d'un network psychiatrique. Ensuite, nous allons détailler les différents processus d'estimation d'un network psychiatrique. Troisièmement, nous allons détailler les différentes mesures utilisées pour interpréter les résultats d'un network, ainsi que leur stabilité et précision. Enfin, nous allons discuter des principales applications que cette approche pourrait développer en pratique clinique courante. Les principales critiques émises à l'égard de l'approche des networks seront intégrées au fur et à mesure que les différentes méthodologies sont introduites.

\section{Structure et composition d'un network psychiatrique}

\section{1 Éléments constituant un network}

Un network est constitué d'un ensemble de noeuds, reliés par un ensemble de bords (16).

Un noeud représente une entité mesurée. Dans d'autres domaines, les noeuds représentent des personnes (network sociaux), des gares, ou des villes. Les network peuvent également explorer via la neuroimagerie des régions du cerveau (network neuroanatomiques). En psychiatrie, les noeuds représentent soit des symptômes/signes observés (dans le cadre de troubles mentaux) ou bien des sous-parties de construits mentaux 
Psychiatrie DES "NETWORKS"

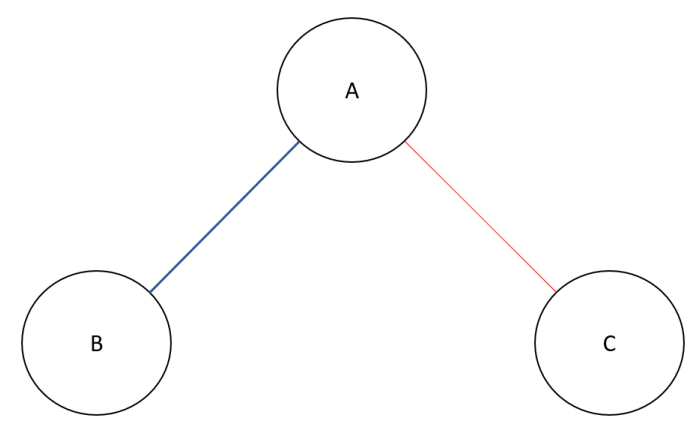

Figure 4 - Un network non dirigé pondéré à trois noeuds (A, B, et $\mathrm{C})$. Les trois noeuds sont connectés par deux bords, A-B, et A-C. A-B dénote une connexion positive; A-C représente une connexion négative.

(tels que l'empathie, le narcissisme, ou la résilience). En visualisation graphique, les noeuds sont représentés par des cercles (le plus souvent), des carrés ou encore des triangles.

Un bord représente une connexion (ou l'absence de connexion, selon le modèle de network qui est choisi) entre deux noeuds. Le plus souvent, un bord est interprété comme la présence (ou l'absence) d'interaction, ou de morbidité, ou bien de causalité entre deux entités. L'interprétation du bord dépend du modèle de network qui est estimé ou visualisé. Par exemple, si nous choisissons d'explorer le network ferroviaire d'une région, un bord va représenter le chemin de fer qui relie deux gares. De même, un network social utilisera les bords pour indiquer une relation mutuelle entre deux personnes. Les bords peuvent être observés (l'observateur sait à priori que deux noeuds sont connectés) ou bien non observés (la présence ou l'absence de relation entre deux noeuds doit donc être testée, en définissant une hypothèse nulle et une hypothèse alternative).

\subsection{Types de networks}

Il existe différents types de network. Nous allons ci-dessous détailler les types de networks les plus utilisés en psychiatrie.

\subsubsection{Network non dirigé, pondéré ou non pondéré}

Un network non dirigé est une structure présentant des noeuds connectés par des bords dont le sens n'est pas connu. Un exemple de network non dirigé est illustré en figure 3 Le bord A-B connectant les noeuds A et B, pourrait, dans un network non dirigé, assumer les 3 sens possibles : A vers B, B vers A, ou symétriquement de A vers B et vice-versa. Les bords d'un network non dirigé peuvent présenter un poids, reflétant l'importance relative d'un bord par rapport à d'autres. Le poids du bord est représenté le plus souvent par un lien plus épais. Les networks contenant des poids sont appelés des networks pondérés; les networks sans poids sont non pondérés.

Pour comprendre l'importance de la pondération, prenons l'exemple de trois symptômes connus de la dépression (17) qui pourraient sous-tendre la figure 3: l'insomnie (A), la fatigue (B), et l'idéation suicidaire (C). L'insomnie pourrait être liée à l'idéation suicidaire ainsi qu'à la fatigue, mais sa connexion à la fatigue est bien plus importante d'un plan clinique qu'à l'idéation suicidaire. Les poids des bords servent précisément pour exprimer cette différence entre les connexions au sein d'un network.

Dans les network pondérés, le poids des bords peut être positif (dénotant une association positive entre deux noeuds) ou négatif (représentant une association négative). La figure 4 montre un network présentant une association positive (A-B) et une association négative (A-C).

Les networks pondérés non dirigés sont les networks les plus utilisés en recherche clinique et méthodologique (18). Les bords représentent le plus souvent des corrélations partielles.

\subsubsection{Network dirigé, cyclique ou acyclique}

Certains travaux utilisent des méthodes statistiques avancées pour déterminer une relation de cause à effet entre deux noeuds (19), et ce même dans des données transversales, grâce à des méthodes de machine learning 


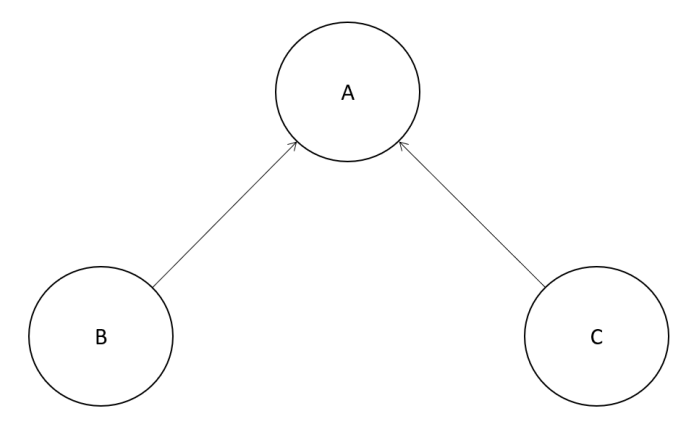

Figure 5 - Un network dirigé acyclique composé de trois noeuds A, B et C. B et C "causent" A. Il s'agit d'une situation de "collision"

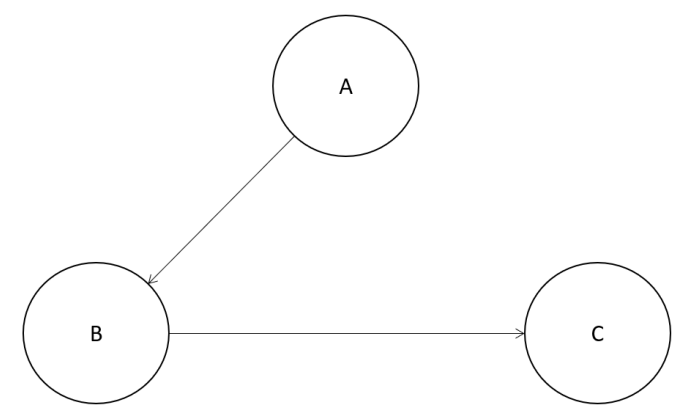

FiguRE 6 - Un network dirigé acyclique composé de trois noeuds A, B et C. A "cause" B, et B "cause" C.

(20). Ces méthodologies utilisent des networks dits "dirigés" puisque le sens des bords est déterminé (21). Il existe deux types de networks dirigés : cycliques ou acycliques. Malgré que leur utilisation soit moins populaire que les networks non dirigés, il est important de connaître les structures de base des networks dirigés puisqu'une structure dirigée pourrait être la "vérité fondamentale" qui sous-tendent certaines symptômes de troubles mentaux. Si sous-jacentes lors de l'analyse d'un network non dirigés, des networks dirigés peuvent générer des résultats inattendus et pouvant être difficilement interprétés.

Deux exemples de networks dirigés acycliques sont proposés dans les figures 5 et 6 .

Dans la figure 5, deux noeuds B et C causent le noeud A. Cette structure est aussi connue sous le nom de "structure en V" et constitue la base pour l'apprentissage automatique des algorithmes qui vont découvrir la structure que sous-tendent les données analysées (20). La structure collision est utile puisqu'elle stocke une information temporelle à un instant donné. Par exemple, si les noeuds $\mathrm{B}$ et $\mathrm{C}$ représentent deux policiers pointant une arme contre un criminel (le noeud A), nous pouvons, en déterminant le statut de A $(0=$ mort, $1=$ vivant $)$, et d'un des deux policiers $(0=$ n'a pas tiré, $1=$ a tiré $)$, inférer le statut de l'autre policier.

Dans la figure 6, A cause B qui à son tour cause C. C ne cause pas A, ce qui laisse la boucle ouverte.

La boucle est par contre fermée dans la figure 7, représentant un network dirigé cyclique. Les networks cycliques sont le plus souvent rencontrés dans les séries temporelles, ou des mesures répétés de paramètres psychiatriques sont effectués pour contrôler l'état mental. Les networks dirigés cycliques ont pu être utiles afin de montrer l'existence d'un "ralentissement critique" des symptômes de dépression : lors de la dégradation d'un état mental, la prédiction entre les symptômes dans un network se concrétise davantage (22).

\section{Estimation d'un network psychiatrique}

\subsection{Dépendance et indépendance conditionnelle}

La présence d'un bord dans un network peut signifier la présence ou l'absence d'une connexion entre deux noeuds. Ceci dépend de si l'estimation d'un network vise à détecter la présence d'une dépendance 
Psychiatrie DES "NETWORKS"

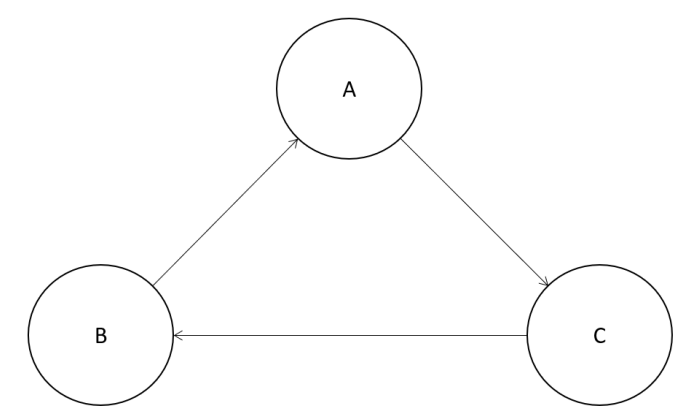

Figure 7 - Un network dirigé cyclique composé de trois noeuds A, B et C. A cause B, B cause C, C cause A.

conditionnelle ou d'une indépendance conditionnelle (23). Une dépendance conditionnelle est comprise comme une association entre deux variables lorsqu'une troisième variable est fixée. Une indépendance conditionnelle est comprise comme l'absence d'association entre deux variable lorsqu'une troisième variable est fixées à des niveaux déterminés (14).

\subsection{Corrélations partielles et leur interprétation}

Le plus souvent, les bords entre les noeuds sont estimés sous forme de corrélation partielle, la traduction statistique du concept théorique de relation de dépendance conditionnelle (18). Un network de corrélations partielles ressemblera aux modèles proposés dans les figures 2 et 4 : un network pondéré non dirigé. Les corrélations partielles seront positives ou négatives, traduisant une association y correspondant entre les noeuds.

Comment interpréter la présence d'une corrélation partielle positive entre deux variables X et Y se trouvant dans un network de symptômes psychiatriques tels que récoltés avec des échelles auto-administrées? D’un point de vue statistique, nous pouvons affirmer que, si une corrélation partielle est présente entre X et $\mathrm{Y}$, cela implique que, en contrôlant toutes les autres variables du network, une connexion existe entre les deux noeuds (7).

Pour translater cette explication mathématique en pratique clinique, une possible interprétation est la suivante : en contrôlant tous les autres symptômes, si X et $\mathrm{Y}$ partagent une connexion, ceci veut dire que dans l'échantillon observé la réponse moyenne du groupe observé à la question $\mathrm{X}$ pourra prédire celle de $\mathrm{Y}$ et vice-versa, puisque le network est non dirigée (11). En pratique, si nous observons une corrélation partielle entre deux symptômes insomnie et fatigue, nous pourrons déduire que si le groupe observé présente en moyenne une insomnie importante, il présentera également une fatigue importante, en contrôlant les niveaux des autres symptômes du network.

\subsection{Modèle Graphique Gaussien, Modèle de Ising, Modèle Graphique Mixte}

Toutes les corrélations partielles d'un network sont déterminées en même temps par l'estimation d'un Modèle Graphique Gaussien (GGM), applicable pour les données continues (ou assimilables à des continues) (24). Le GGM (le network lui-même) est estimée comme l'inverse de la matrice de covariance dérivée de la base de données de symptômes recueillie. L'alternative pour variable binaire du GGM est le modèle de Ising, dérivant de la physique statistique et adapté en psychiatrie (25), mais rarement utilisé lors de travaux empiriques (8). Selon le type de variables inclues (par exemple, si une variable binaire telle le sexe des participants est rajoutée dans une base de données continue), des Modèles Graphiques Mixtes (MGM) peuvent être utilisés (26). Le GGM est néanmoins le modèle utilisé de façon prédominante dans la littérature.

Le GGM est facile à comprendre, surtout s'il est utilisé pour étudier un trouble mental. Soit $\mathbf{y}=\left(y_{1}, \ldots, y_{p}\right)^{\top}$ un vecteur multivarié normal quelconque. Sans perte d'information, les données sont considérées comme centrées avec un vecteur moyen de 0 et une matrice de covariance $\Sigma$. L'inverse de la matrice de covariance $\Theta=\Sigma^{-1}$ est ciblée dans l'analyse des networks, puisqu'en standardisant les éléments ne se trouvant pas sur la diagonale de la matrice nous retrouvons les coefficients de corrélations partielles $(\rho)$. Le GGM associé avec $\mathbf{y}$ est un netwok non dirigé qui est dénoté habituellement $G=(V, E)$. Il inclut un set de noeuds $V=\left\{y_{1}, \ldots, y_{p}\right\}$ qui correspond à, disons, un groupe de symptômes dans les questionnaires, ainsi qu'à un set de bords $E$ qui 
inclut les "connexions". Dès lors, il existe un bord entre deux noeuds $y_{i}$ and $y_{j}$ quand ils sont déterminés comme conditionnellement dépendants (par exemple, $\rho_{i j} \neq 0$ ).

\subsection{La régularisation, ses bénéfices et ses dangers}

Dans la vaste majorité des networks de troubles mentaux étudiés à ce jour, le GGM subit un processus de régularisation- $\ell_{1}$ associé à un EBIC (Critère Bayésien Étendu d'Information) appelé "lasso" (acronyme pour "least absolute shrinkage and selection operator"), qui "calibre" le network réduit les petites corrélations partielles à zéro; il rend dès lors un modèle "conservateur" (avec le moins de connexions possible) (18), considéré plus facile à visualiser et interpréter. Néanmoins, de récents travaux ont montré que ce processus statistique n'est pas sans inconvénients (23) : nous allons ici en lister les deux plus importantes.

Premièrement, la régularisation a été définie comme inconsistante pour la sélection des modèles networks, avec un haut risque d'obtention de faux positifs dans des cas à "haute dimension", c'est à dire quand la taille de l'échantillon dépasse grandement le nombre de variables. Ce cas de figure est hautement représenté dans les études psychiatriques, ce qui limite le potentiel de la régularisation (27); néanmoins, depuis l'expression de cette première critique, la méthodologie de la régularisation a introduit l'utilisation d'une "valeur seuil" pour l'inclusion des bords dans le network, ce qui a pu limiter le taux de faux positives dans de publications récentes (10). Deuxièmement, la régularisation ne fournit pas de preuve en regard de l'absence de connexion entre deux symptômes (hypothèse nulle) : dans un network estimé via régularisation, dès lors, l'absence de connexion n'est pas prouvée. Cette dernière conséquence est fort importante, puisque la détection des relations d'indépendances conditionnelles entre variables d'un modèle est une des idées de base de l'approche network.

\subsection{Estimation Bayésienne du GGM}

Il est important de noter que la présence d'un zéro dans la matrice d'un network correspond à une corrélation partielle de zéro, et ne prouve donc pas l'existence d'une relation d'indépendance conditionnelle entre les variables (symptômes) partageant la corrélation partielle de zéro; ce qui implique que paradoxalement, l'absence d'un bord entre deux noeuds d'un network estimé via un GGM classique ne peut pas confirmer l'absence de connexion.

Pour pallier les problèmes de la régularisation et de l'absence de méthodologie pouvant tester formellement la présence d'une relation de dépendance indépendance conditionnelle, une méthode d'estimation Bayésienne du GGM ("BGGM") a été récemment introduite (24). Cette méthodologie utilise le facteur de Bayes (reflétant directement un niveau de preuve) comme seuil de détection de relations de dépendance conditionnelle/inconditionnelle dans un network, permet la détermination du GGM sur base des probabilités à posteriori (en tenant donc compte des données), et offre la possibilité au chercheur de tester formellement les relations conditionnelles de dépendance ou d'indépendance au sein d'un network. La plus-value de cette approche est de fournir une information quant au niveau de preuve à disposition en faveur de l'hypothèse testée ou de l'exploration du network sans hypothèse à priori.

BBGM, malgré son introduction récente, a pu montrer des avantages dans une réanalyse de network (28), et des études sont en cours pour la tester de façon empirique.

\section{Inférence du network psychiatrique}

\subsection{Centralité et rédondance}

La centralité est une mesure importée, comme le reste de la technique des networks, de la physique statistique : elle sert à représenter l'importance relative d'un symptôme par rapport aux autres symptômes du network. La mesure la plus utilisée pour reporter la centralité d'un symptôme est la force. La force est définie comme la somme des valeurs absolues des bords qu'un symptôme donné partage avec d'autres symptôme dans un network (16). Parce que certains networks présentent des bords négatifs, une autre mesure de centralité, tenant compte de la somme des valeurs relatives des bords; cette mesure est connue sous le nom de influence attendue (11).

Un problème est remarqué avec l'utilisation de la centralité lors de l'application des networks aux troubles mentaux tels que mesurés dans des échelles; en effet, la plupart des échelles contiennent des mesures répétées du même type de symptôme, ce qui implique la présence de noeuds redondant au sein du même network. Lorsque cela arrive, les noeuds redondants sont fortement connectés, ce qui hausse considérablement leur 


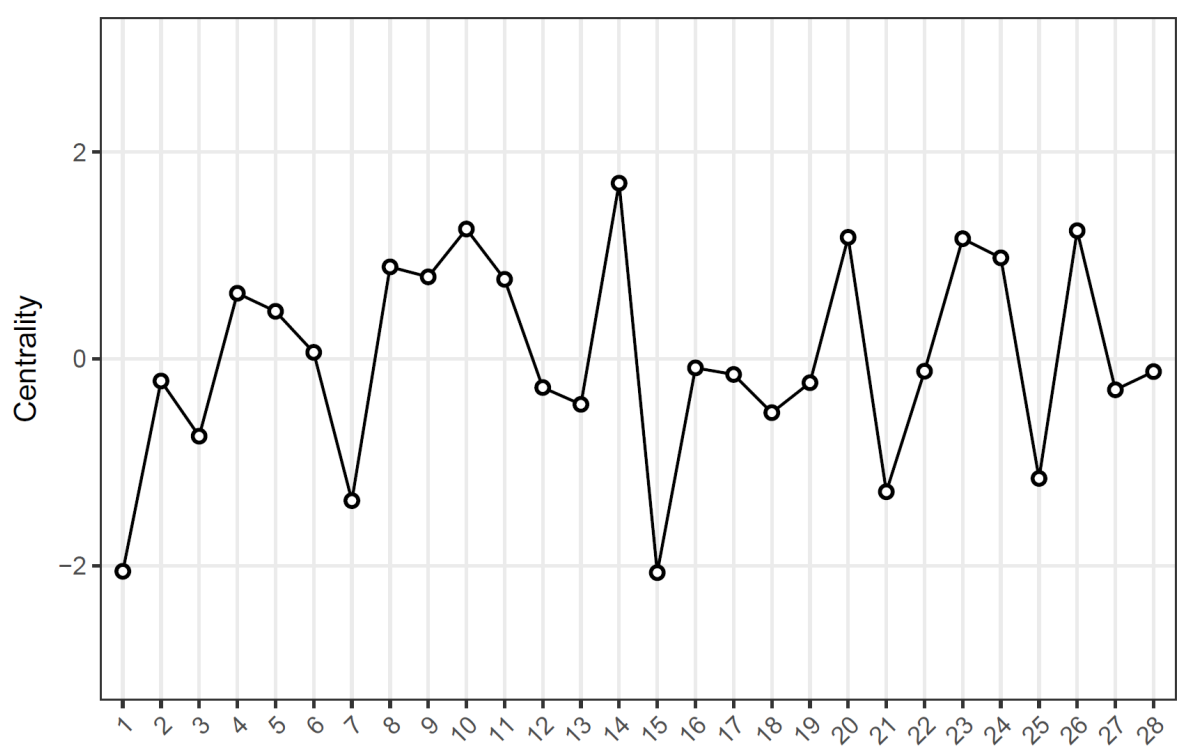

Figure 8 - Un graphique associant à chaque noeud une valeur de centralité (scores z standardisés). Graphique dérivé de Briganti et al., 2018 (7)

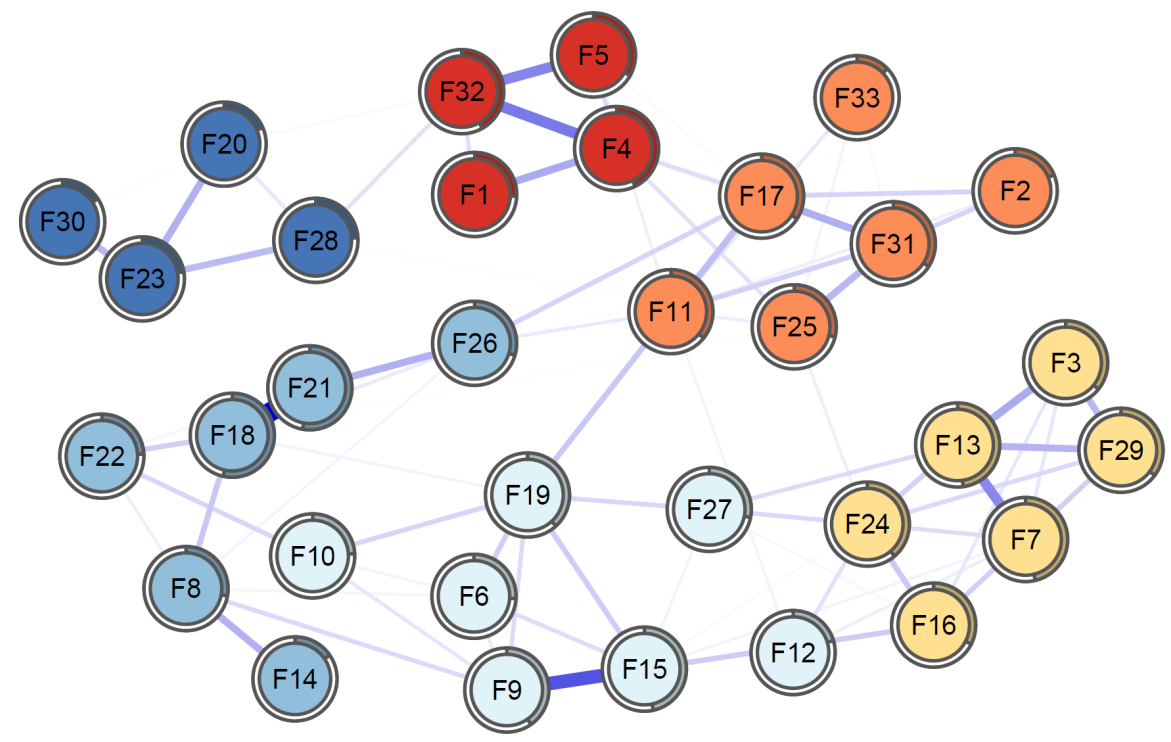

Figure 9 - Un network présentant des noeuds entourés de leur prédictibilité respective. Graphique dérivé de Briganti et Linkowski, 2019 (13)

centralité, mais au coût d'un changement de la nature de leur connexion : cette dernière devra donc être interprétée comme variance partagée entre les deux symptômes, ce qui fausse l'interprétation des indices de centralité du network (13). Un autre point faible de cette technique est la relativité de l'indice estimé : au sein d'un network, il y a aura toujours un symptôme plus central qu'un autre, même si le symptôme le plus central présente en réalité des indicateurs faibles.

Un exemple de graphique de centralité est retrouvé en figure 8 . 


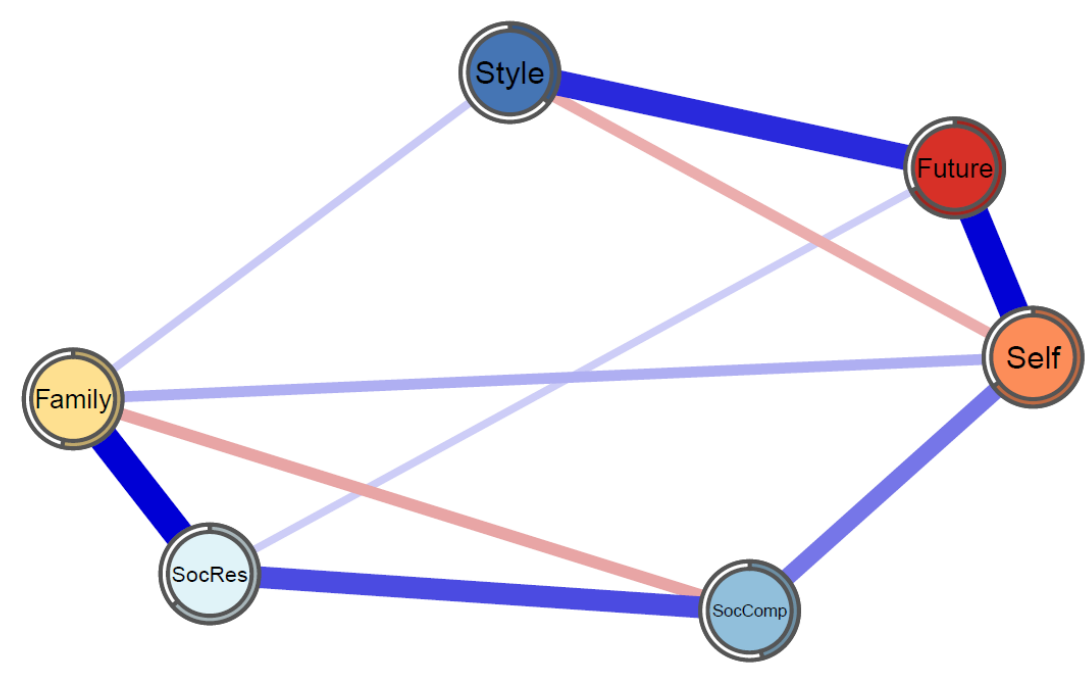

FiguRe 10 - Un network de domaines rassemblant les noeuds de la figure 9. Graphique dérivé de Briganti et Linkowski, 2019 (13)

\subsection{Prédiction entre symptômes}

La prédictibilité des noeuds a été introduite comme une mesure de centralité alternative se voulant une mesure absolue de la connectivité d'un symptôme dans un network. La prédictibilité des noeuds représente la variance partagée d'un noeud donné avec les noeuds environnants (29). Le plus souvent, la prédictibilité des noeuds est représentée par un diagramme circulaire rempli à hauteur des pourcentage, comme illustré en figure 9. la prédictibilité des noeuds n'échappe pas au problème de la redondance des symptômes, puisque si deux symptômes se ressemblent, ils seront hautement prédictibles dans un network. Des méthodes bayésiennes sont maintenant également applicables au calcul de ce paramètre (30). La prédictibilité des noeuds a été définie comme la limite supérieure de la controlabilité : si un noeud A est hautement prédictible et nous considérons que tous les bords qu'il partage avec d'autres noeuds sont dirigés vers A, alors nous pouvons considérer que la prédictibilité est le réflet du contrôle que les autres variables ont sur le noeud A (11).

\subsection{Détection de communautés dans un network}

Pouvoir structurer les symptômes ou les construits mentaux en domaines (ou dimensions) pouvant expliquer leur cohésion clinique d'un point de vue empirique est un défi de recherche de longue haleine en psychiatrie (5). La psychiatrie des networks possède ses propres outils de détection de domaines, comme l'algorithme walktrap dérivé du machine learning et utilisé pour explorer des dimensions (7); cet algorithme se base sur la distance computée entre symptômes pour établir des regroupements : les symptômes qui sont "proches" tendent en effet à former une communauté dans le network. Il a été montré que cet algorithme possède une bonne stabilité pour retrouver le bon nombre de communauté en se basant sur de larges données ; il a dès lors inspiré la création de l'analyse graphique exploratoire (15), utilisée comme approche systématisée en psychiatrie des networks pour vérifier si le nombre prévu de domaines d'un construit ou trouble mental est retrouvé dans un échantillon donné. Cette méthodologie a permis de montrer que, dans certains cas, un ou plusieurs domaines peuvent être redondants dans un trouble mental, et que les fusionner permet d'obtenir des résultats plus pertinents aux données $(13,10)$.

\subsection{Networks de domaines psychiatriques}

Nous avons pu montrer comment l'analyse network d'échelles représentant un construit mental rapporte souvent de communautés correspondantes aux facteurs originellement proposés (7) 11). Bien que les interactions entre symptômes/items d'une même communauté soit intéressante, l'analyse network dévoile sa vraie valeur ajoutée lorsqu'on regarde aux interaction entre symptômes de communautés différentes, afin de montrer le comportement de plusieurs facettes des construits ou troubles mentaux. 
C'est dans ce cas que la psychométrie network généralisée a été développée (14); cette approche permet de combiner l'approche factorielle (regroupant le plus de variables possibles sous la même étiquette), puis d'analyser les connexions entre les variables restantes (c'est à dire correspondant véritablement à un network de composantes à part entière). Nous avons pu montrer que cette approche porte ses fruits lorsqu'on s'attaque à des construits mentaux ou plusieurs noeuds font référence au même aspect de comportement pour mettre en évidence des relations positives ou négatives entre domaines (13).

\section{$5 \quad$ Stabilité et précision dans l'estimation des networks}

Très rapidement la question de la stabilité et la précision des résultats obtenus dans l'estimation des network psychiatriques et devenue importante dans ce nouveau domaine de recherche. Les networks ont montré des résultats similaires dans plusieurs populations dans le cadre du syndrome du stress post-traumatique (6) ce qui rajoute un argument de soutien pour la réplication des résultats obtenus via ces analyses.

L'instrument le plus utilisé pour analyser la stabilité et précision des paramètres des networks est le bootstrapping (18). Cette méthode ré-calcule les paramètres qui constituent le network pour répondre à des questions déterminées. Pour ce qui concerne les bords, les 2 questions sont les suivantes : 1) est-ce que mon bord A entre deux noeuds $\mathrm{X}$ et $\mathrm{Y}$ est vraiment plus grand que mon bord $\mathrm{B}$ entre deux noeuds $\mathrm{V}$ et $\mathrm{W}$ ? et 2) est-ce que mon bord A est correctement estimé?

Pour répondre à la première question, des intervalles de confiance sont estimés autour de la différence entre les bords $\mathrm{A}$ et $\mathrm{B}$ tels que déterminés par un nombre de ré-estimations au choix ; généralement, 2000 ré-estimations du network sont performées (7), si ce test se révèle positif, l'observateur peut interpréter qu'un bord A est significativement plus grand qu'un bord B (11). Pour répondre à la deuxième question, des intervalles de confiance sont calculés autour de plusieurs milliers de ré-estimations de tous les bords du network. Si ces intervalles de confiance sont peu larges et ne se chevauchent pas entre différents bords, alors l'observateur peut mieux apprécier la différence entre différents bords du network (visualisés sous forme d'épaisseurs en figure 2

Le même procédé peut être appliqué pour les indicateurs de centralité.

\section{Discussion : applications courantes et futures de la psychiatrie des "networks" en pratique clinique}

Cet article avait comme but de se focaliser sur l'important sujet de la psychiatrie des networks, permise par le développement de la théorie network des troubles mentaux (5). Nous avons introduit les problématiques actuelles dans l'étude et classification actuelle des construits et troubles mentaux, pour lesquels l'analyse des networks est posée comme une alternative intéressante et nouvelle. Ensuite, nous avons introduit l'état de l'art sur la théorie et l'analyse des networks telle que exploitée pour la grande majorité des études dans la littérature ainsi que les défauts et critiques principales exposées pour les méthodes.

Lorsque une grande partie de construits et troubles mentaux ont été redéfinis grâce à l'analyse des networks (6) 8, 13; 21), les prochaines étapes du développement de cette nouvelle science se dessinent : nous allons discuter de quatre parmi celles-ci.

Premièrement, analyser l'inférence du network s'est révélée utile pour prédire les outcomes post-traitement dans le cas de l'anorexie (31) : ceci reflète l'utilité potentielle de mesurer les symptômes centraux dans un network et s'en servir afin de formuler et tester des hypothèses formelles à caractère clinique.

Deuxièmement, l'analyse des networks prend tout son sens lorsqu'elle sert à redéfinir la théorie psychiatrique sous-tendant les troubles mentaux : c'est ainsi que par exemple, de nouveaux modèles computationnels du "trouble de panique" a été développé récemment (32) ; comme ce dernier, une large partie des troubles mentaux pourrait être redéfinie grâce à l'analyse des networks, ce qui permettra dans le futur la naissance de nouvelles classifications correspondant davantage aux avancées effectuées en recherche tant fondamentale que clinique.

Troisièmement, nous assistons à l'émergence de networks "hybrides" mélangeant des symptômes et les régions du cerveau dans de récents travaux (33); dans le futur, nous pourrons étendre l'approche des networks aux différents niveaux déterminant le comportement humain normal et anormal, pour ainsi avoir une vue étendue sur les dynamiques contrôlant les construits et troubles mentaux tant à un niveau symptomatique qu'électrophysiologique, neuroanatomique, biologique et génétique. 
Quatrièmement, parce que l'approche des networks reconsidère le symptôme comme composante active dans le trouble mental, une approche interventionnelle est préconisée afin de monitorer comment des interventions thérapeutiques affectent des symptômes donnés et leurs relations dans le network : cette approche, testée récemment, a été définie comme l' "analyse interventionnelle des networks" (34). L'analyse des networks se propose donc également comme un outil de monitoring objectif des thérapies en psychiatrie, une perspective fort intéressante d'un point de vue clinique.

Bien que relativement "jeune" (le domaine de recherche est né il y a environ dix ans), la psychiatrie des networks a été rapidement transformée d'un outil de recherche fondamentale à une technique appliquée à la pratique clinique. Les perspectives d'études futures sont imprégnées d'un intérêt translationnel nécessaire à l'amélioration des pratiques en psychiatrie.

\section{Références}

[1] American Psychiatric Association. Diagnostic and Statistical Manual of Mental Disorders. 5th ed. American Psychiatric Association; 2013.

[2] Borsboom D, Cramer AOJ. Network Analysis : An Integrative Approach to the Structure of Psychopathology. Annual Review of Clinical Psychology. 2013 Mar;9(1) :91-121. Available from : http://www. annualreviews.org/doi/10.1146/annurev-clinpsy-050212-185608.

[3] Marsman M, Borsboom D, Kruis J, Epskamp S, Bork Rv, Waldorp LJ, et al. An Introduction to Network Psychometrics : Relating Ising Network Models to Item Response Theory Models. Multivariate Behavioral Research. 2018 Jan ;53(1):15-35. Available from : https://doi.org/10.1080/00273171.2017.1379379.

[4] Borsboom D. Psychometric perspectives on diagnostic systems. Journal of Clinical Psychology. 2008 Sep ;64(9) :1089-1108.

[5] Borsboom D. A network theory of mental disorders. World Psychiatry. 2017 Feb ;16(1) :5-13. Available from : https://www.ncbi.nlm.nih.gov/pmc/articles/PMC5269502/

[6] Fried EI, Eidhof MB, Palic S, Costantini G, Huisman-van Dijk HM, Bockting CLH, et al. Replicability and Generalizability of Posttraumatic Stress Disorder (PTSD) Networks : A Cross-Cultural Multisite Study of PTSD Symptoms in Four Trauma Patient Samples. Clinical Psychological Science. 2018 ;Available from : https://doi.org/10.1177/2167702617745092.

[7] Briganti G, Kempenaers C, Braun S, Fried EI, Linkowski P. Network analysis of empathy items from the interpersonal reactivity index in 1973 young adults. Psychiatry Research. 2018 Jul;265:87-92. Available from : http://www.sciencedirect.com/science/article/pii/S0165178117316827

[8] Briganti G, Linkowski P. Exploring network structure and central items of the Narcissistic Personality Inventory. International Journal of Methods in Psychiatric Research. 2019 ;n/a :e1810. Available from : https://onlinelibrary.wiley.com/doi/abs/10.1002/mpr.1810.

[9] Mullarkey MC, Marchetti I, Beevers CG. Using Network Analysis to Identify Central Symptoms of Adolescent Depression. Journal of Clinical Child \& Adolescent Psychology. 2018 Mar;0(0):1-13. Available from : https://doi.org/10.1080/15374416.2018.1437735.

[10] Briganti G, Linkowski P. Network Approach to Items and Domains From the Toronto Alexithymia Scale. Psychological Reports. 2019 Nov ;p. 0033294119889586.

[11] Briganti G, Fried EI, Linkowski P. Network analysis of Contingencies of Self-Worth Scale in 680 university students. Psychiatry Research. $2019 ; 272: 252-257$.

[12] Deserno MK, Borsboom D, Begeer S, Geurts HM. Multicausal systems ask for multicausal approaches : A network perspective on subjective well-being in individuals with autism spectrum disorder. Autism. $2017 ; 21(8): 960-971$.

[13] Briganti G, Linkowski P. Item and domain network structures of the Resilience Scale for Adults in 675 university students. Epidemiology and Psychiatric Sciences. 2019 ;p. 1-9.

[14] Epskamp S, Rhemtulla M, Borsboom D. Generalized Network Psychometrics : Combining Network and Latent Variable Models. Psychometrika. 2017 Dec;82(4):904-927. Available from : https://doi.org/ 10.1007/s11336-017-9557-x

[15] Golino HF, Epskamp S. Exploratory graph analysis : A new approach for estimating the number of dimensions in psychological research. PLOS ONE. 2017 Jun;12(6) :e0174035. Available from : http://journals.plos.org/plosone/article?id=10.1371/journal.pone.0174035. 
[16] Boccaletti S, Latora V, Moreno Y, Chavez M, Hwang DU. Complex networks : Structure and dynamics. Physics Reports. 2006 Feb ;424(4):175-308. Available from : http://www. sciencedirect.com/science/ article/pii/S037015730500462X.

[17] Zung WW. A Self-Rating Depression Scale. Archives of General Psychiatry. 1965 Jan;12:63-70.

[18] Epskamp S, Fried EI. A tutorial on regularized partial correlation networks. Psychological Methods. 2018 Dec ;23(4):617-634.

[19] Moffa G, Catone G, Kuipers J, Kuipers E, Freeman D, Marwaha S, et al. Using Directed Acyclic Graphs in Epidemiological Research in Psychosis : An Analysis of the Role of Bullying in Psychosis. Schizophrenia Bulletin. 2017 Oct;43(6):1273-1279. Available from : https://academic.oup.com/ schizophreniabulletin/article/43/6/1273/3829522.

[20] Scutari M. Learning Bayesian Networks with the bnlearn R Package. Journal of Statistical Software. $2010 \mathrm{Jul} ; 35(1): 1-22$. Available from : https://www.jstatsoft.org/index.php/jss/article/view/ v035i03.

[21] Briganti G, Scutari M, Linkowski P. A Bayesian Network approach to symptoms from the Zung Depression Scale. 2020 ;.

[22] Wichers M, Groot PC. Critical Slowing Down as a Personalized Early Warning Signal for Depression. Psychotherapy and Psychosomatics. $2016 ; 85(2): 114-116$. Available from : https://www.karger.com/ DOI/10.1159/000441458.

[23] Williams DR, Rhemtulla M, Wysocki AC, Rast P. On Nonregularized Estimation of Psychological Networks. Multivariate behavioral research. $2019 ; 54(5): 719-750$. Available from : http://europepmc. org/abstract/med/30957629.

[24] Williams DR. Bayesian Estimation for Gaussian Graphical Models : Structure Learning, Predictability, and Network Comparisons. 2018 Sep;.

[25] van Borkulo CD, Borsboom D, Epskamp S, Blanken TF, Boschloo L, Schoevers RA, et al. A new method for constructing networks from binary data. Scientific Reports. 2014 Aug; 4 :5918. Available from : https://www.nature.com/articles/srep05918

[26] Haslbeck JMB, Waldorp LJ. mgm : Structure Estimation for Time-Varying Mixed Graphical Models in high-dimensional Data. arXiv preprint :151006871v2. 2016 ;Available from : http://arxiv.org/abs/ $1510.06871 \mathrm{v} 2$.

[27] Williams DR, Mulder J. Bayesian Hypothesis Testing for Gaussian Graphical Models : Conditional Independence and Order Constraints. 2019 Jan;Available from : https://osf .io/ypxd8

[28] Williams DR, Briganti G, Linkowski P, Mulder J, Rhemtulla M. On Testing the Null Hypothesis of Conditional Independence : A Bayesian Reanalysis of Four Psychological Networks. 2020 ;:

[29] Haslbeck JMB, Fried EI. How predictable are symptoms in psychopathological networks? A reanalysis of 18 published datasets. Psychological Medicine. 2017 Dec;47(16):2767-2776.

[30] Williams DR, Mulder J. BGGM : A R Package for Bayesian Gaussian Graphical Models. 2019 Jun;Available from : https://psyarxiv.com/3b5hf/.

[31] Elliott H, Jones PJ, Schmidt U. Central Symptoms Predict Posttreatment Outcomes and Clinical Impairment in Anorexia Nervosa : A Network Analysis. Clinical Psychological Science. 2019 Oct; Available from : https://doi.org/10.1177/2167702619865958.

[32] Robinaugh D, Haslbeck JMB, Waldorp L, Kossakowski JJ, Fried EI, Millner A, et al. Advancing the Network Theory of Mental Disorders : A Computational Model of Panic Disorder. 2019 May ;.

[33] Hilland E, Landrø NI, Kraft B, Tamnes CK, Fried EI, Maglanoc LA, et al. Exploring the Links between Specific Depression Symptoms and Brain Structure : A Network Study. Psychiatry and Clinical Neurosciences;.

[34] Blanken TF, Van Der Zweerde T, Van Straten A, Van Someren EJW, Borsboom D, Lancee J. Introducing Network Intervention Analysis to Investigate Sequential, Symptom-Specific Treatment Effects : A Demonstration in Co-Occurring Insomnia and Depression. Psychotherapy and Psychosomatics. 2019 Mar;88(1):52-54. Available from : https://www.ncbi.nlm.nih.gov/pmc/articles/PMC6469840/ 